\title{
The aging revolution
}

The aging of the population brings with it major changes in the relative importance of illnesses. From the situation that prevailed in the last century, when acute illnesses were preponderant, we are now moving to an era when chronic diseases are becoming increasingly prevalent. The hospital-centered healthcare system of the 19th and 20th centuries that was created to deal with acute and especially infectious diseases is inappropriate for responding to the long-term, ongoing treatment needs of the chronically ill. We must revamp the healthcare system and centre it on the place where people live, i.e., their home. The health care system should thus shift from a hospital-centered to a community-centered model.

Since most chronic diseases are preventable, health promotion and disease prevention should be prioritized. Promoting healthy life habits is critical to decrease the future burden of chronic diseases. Tobacco cessation, healthy nutrition, regular physical exercise and good sexual habits are key issues. But health promotion should go beyond in targeting also lodging, employment, education, revenue and environmental issues which are strong determinants of health.

Primary care is essential to ensure adequate follow-up of patients with chronic diseases to monitor disease evolution, adjust treatment, and prevent deterioration and undue emergency room and hospital utilization. Physicians and other health professionals should be supported by geriatric teams to provide specialized care to the frail older people suffering from functional decline and acute deterioration. Geriatric services must be available not only in institutions (geriatric assessment unit, rehabilitation unit), but also in the community (day hospital, day center, outpatient clinic, memory clinic).

Patient engagement in their own care is part of the Chronic care model aiming to improve the productive interactions between a well-informed and activated patient with a prepared pro-active inter professional team. The era of almighty physicians prescribing to passive patients is over. Physicians should now work with other professionals; patients together with their family should take part in the decisions and be responsible for their own health.

The care and services required by people suffering from chronic diseases go beyond medical treatment, which will consequently have a more marginal role to play. People's disabilities due to chronic illnesses are now the main driver for health care and services utilization. Our objective should be to optimize the autonomy of individuals by reducing their disabilities and supplying the support needed to mitigate them.

Current healthcare systems area long way from completing this refocusing process. Access to coordinated services is lacking. Integration of health and social services and coordinating the multiple providers involved in care of older frail people are important challenges. Models of full integration like PACE in the USA or coordination like PRISMA which we developed, validated and implemented in Quebec are proven solutions to improve continuity of care.

Home care is clearly insufficient, with informal caregivers who should be indispensable partners in the health care system being more or less left to themselves, with neither support nor respite. Without proper care at home, seniors and their families are given only one option: institutionalization. This costly and unwarranted solution comes with more than its share of problems, such as depersonalization and cramped conditions, break-up of the social network, transmission of infections, separation of couples, and difficulties in recreating a satisfactory living environment. There is an urgent need to change our approach and offer services where they are needed, irrespective of living environment. Rather than forcing people to go where services are provided, services need to travel to where people live. This kind of approach implies a major investment in supporting people's autonomy and requires the establishment of benefits in cash or in kind based on people's needs, not their living environment. Public long-term care insurance plan have been developed by many countries in Europe and Asia to cover the growing risk of functional decline.

The aging of the population implies a real revolution in healthcare and professional practice. Are we ready to move?

Réjean Hébert

School of Public Health, Université de Montréal 